\title{
Coherently Radiating Periodic Structures (CORPS): A Step TowardS High RESOLUTION IMAGING SYSTEMS?
}

\author{
R. García, D. Betancourt, A. Ibañez and C. del-Río \\ Antenna Group, Universidad Pública de Navarra, \\ Campus Arrosadía s/n, 31006 Pamplona, Spain. \\ Phone: +34 948169326 \\ E_mail: carlos@unavarra.es
}

The coherently radiating periodic structures (CORPS) uses the existing mutual coupling between the elements of an array to spread horizontally and coherently (in-phase) the complex amplitudes of each active element of the array. Despite the elements of the array were not too directive, the corporative behavior of all them, radiating coherently the same information, provides high directive beams arising from each array element, being possible narrow distances between beams.

This concept applied to imaging systems, based on periodic disposition of low-profile radiating elements, could offer high resolution images in the range of microwaves and millimeter waves.

\section{Introduction}

The Coherently Radiating Periodic Structures (CORPS) consists in using the same type and size of radiating elements used in the original array to define a periodic structure surrounding all the radiating elements (figure 1).

To complete the definition of being a CORPS, the coherency in the mutual coupling must be introduced, so the period or lattice of the whole new structure should be tuned to be exactly an effective wavelength of the coupling mechanism between elements. This will help us to isolate two active elements of the original array since a stop-band filter is defined with the periodic structure. The first effect of this filter is to retain the power under the active elements (figure 2).

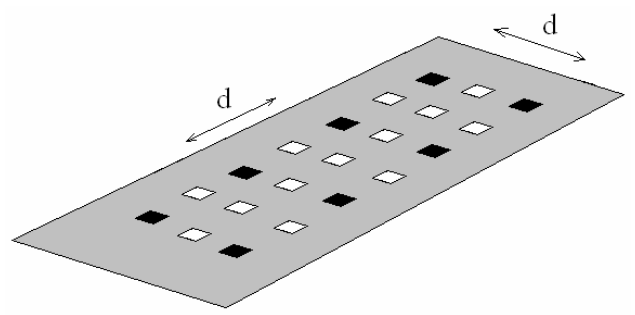

Figure 1.- Coherently Radiating Periodic Structure formed by the active elements (black) and the passive ones (white). The lattice of active elements remains at the same distance, $\boldsymbol{d}$.

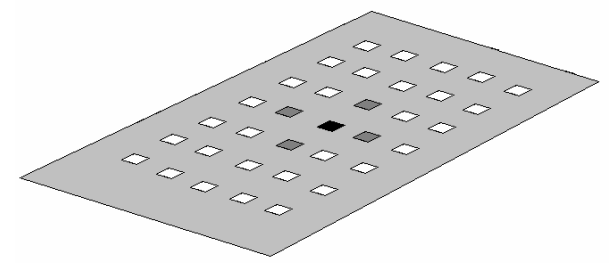

Figure 2.- Coupled elements (dark grey) by one active element (black).

Depending of the type of coupling mechanism and the kind of radiating elements $[1,2]$, some coverage area will be defined surrounding every active element (originally fed). Thus, the residual power coupled to the passive elements will be re-radiated by them, since they are tuned to resonate at the same frequency; but at the same time, the radiating power is added in-phase, since the coherency in the coupling mechanism has been fixed in the definition of the lattice of the periodic structure. 
The immediate consequence is an increase of the radiating effective area of each active element, since the passive elements surrounding it will radiate coherently.

In a practical implementation, the designer could choose to keep the same number of active elements of the array, selecting the dielectric material that allows either the insertion of a desired number of passive elements in between two actives. Other possibility is the reduction of the number of active elements, keeping the overall number of elements but now with some of them being passive and horizontally coupled by the remaining active elements.

For example, in the first case, if we have a classical array with a distance between active elements of $2 / 3 \quad \lambda_{0}$, with a dielectric with a permittivity of 9 we would be able to include a passive element between two active elements and achieve coherent coupling between all the elements, since $\lambda_{\text {dielectric }} \approx 1 / 3 \lambda_{0}$.

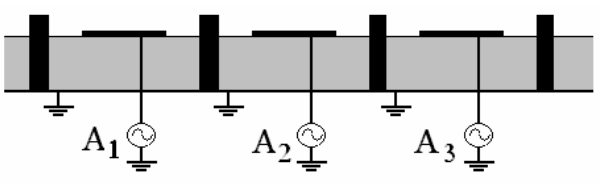

a)

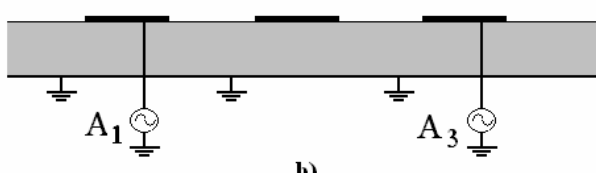

Figure 3.- Linear array of three active self-isolated elements (a) and the same linear array but with non-self-isolated elements with the central excitation removed (b).

The designer could also choose to reduce the number of active elements, reducing at the same time the complexity of the required feeding network, with the penalty of having a reduction in the total power effectively radiated by the structures since the number of input ports is reduced (figure $3)$.

\section{Ideal horizontal coupling mechanism}

As it was established above, the CORPS separates each element of the structure a full effective wavelength. By establishing exactly this distance between elements, a stop-band filter is defined, so no additional problems with the substrate wave modes could be expected since they will be under cut-off conditions. Nevertheless, some residual power will arrive to the neighbouring elements of each active antenna.

For instance, in a rectangular lattice, by leaving a passive element between two active elements, a chess-board-like lattice is defined; where the white spots could be passive elements and the black spot the active ones.

If we were able to couple the half of the power to the neighbouring elements retaining the other half of the power in the active element, the sampled excitation (of an active antenna array) could be reproduced in the radiation layer but with double number of samples.

In this case, every active element is surrounded by four passive one, so every passive element will couple $1 / 8$ of the power originally transferred to the active element. At the same time, every passive element is surrounded by four active elements, so all of them are coupling the same portion of power, obtaining in the end half of the power coupled to every element of the structure.

\section{High resolution imaging systems}

It we could reproduce this phenomena in different stacked layers, creating a 3D structure, figure 4 , we would spread the information of each original active 
element in a wider area of the final radiating layer, getting various highdirectivity beams very close to each other, improving substantially the resolution of imaging systems.

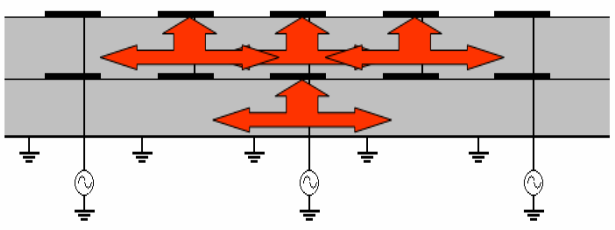

Figure 4.- Side view of a 3D-CORPS of patches, showing the couplings generated only by the central active element between the different elements and layers.

In figure 5, the upper view of the coupled elements after two layers of radiating elements properly matched are drawn, for a rectangular and a triangular lattice. With the triangular lattice, because all distances are equal, the number of coherently coupled elements is higher than with rectangular lattice.

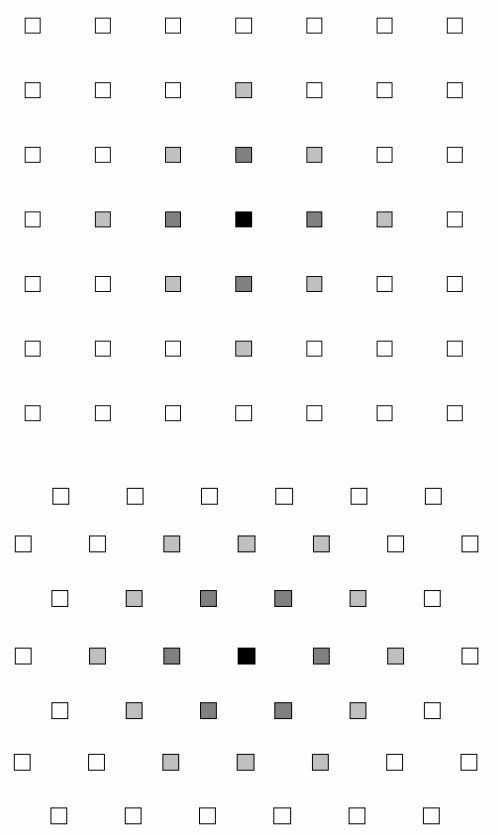

Figure 5.- Upper view of the coupled elements by only one active element after two layers of radiating elements.

\section{Performed simulations}

An example of this kind of 3D-CORPS is represented in figure 6, where two different layers of square patches are separated by other layer of cross-shape slots. The horizontal disposition of the patches and slots horizontally corresponds to a full wavelength, approximately $27 \mathrm{~mm}$, and the distance between layers is defined to be $5 \mathrm{~mm}$. All the elements are designed to resonate at the central frequency of $10 \mathrm{GHz}$.

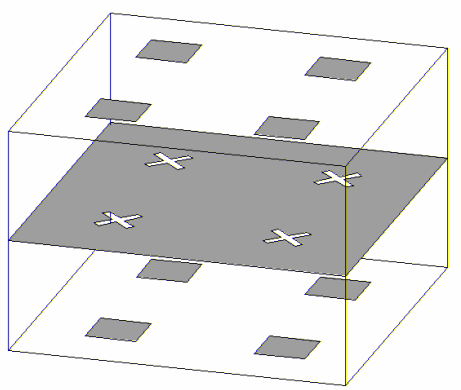

Figure 6.- Four cells of the 3D-CORPS simulated.

This structure shows a vertical bandpass behaviour around $10 \mathrm{GHz}$ in a relatively wide bandwidth.

The performed experiment was to place a elemental dipole antenna $14 \mathrm{~mm}$ from one side of the 3D-CORPS represented in figure 6 but taking into account a square structure of $7 \times 7$ elements. At the other side the re-radiated power was calculated.

The resulting far field pattern (figure 7) shows that the structure reproduces again very accurately the far field pattern of the original dipole. The ripple is due to the limited size of the analyzed structure.

It is important to notice that the structure, as it is represented in figure 6, is rotated 90 degrees and the radiation of the dipole excites the patches of one face (left) of the structure, and the power 
passes through the structure reconstructing at the other side (right) the same field distribution without losing any power.

The coupling mechanism described above ensures that each input patch (over the left face of the structure where the dipole is placed) will couple coherently at least three slots, and these three slots couple five radiating patches, being all of them radiating in phase the complex amplitude coupled to the first patch by the exciting dipole.

\section{Conclusions}

A very promising technology is arising making possible the high resolution imaging systems since it has been demonstrated the possibility to obtain high directive beams quite close to each other.

\section{References:}

[1] Pozar, D.M., "Considerations for millemeter-wave printed antennas." IEEE Trans. Antennas and Propagation, Vol. 31, pp. 740-747, Sept. 1983.

[2] Rebeiz, G.M., "Millimeter-wave and terahertz integrated circuit antennas.", Proc. IEEE, Vol. 80, pp. 1749-1770, Nov. 1992.

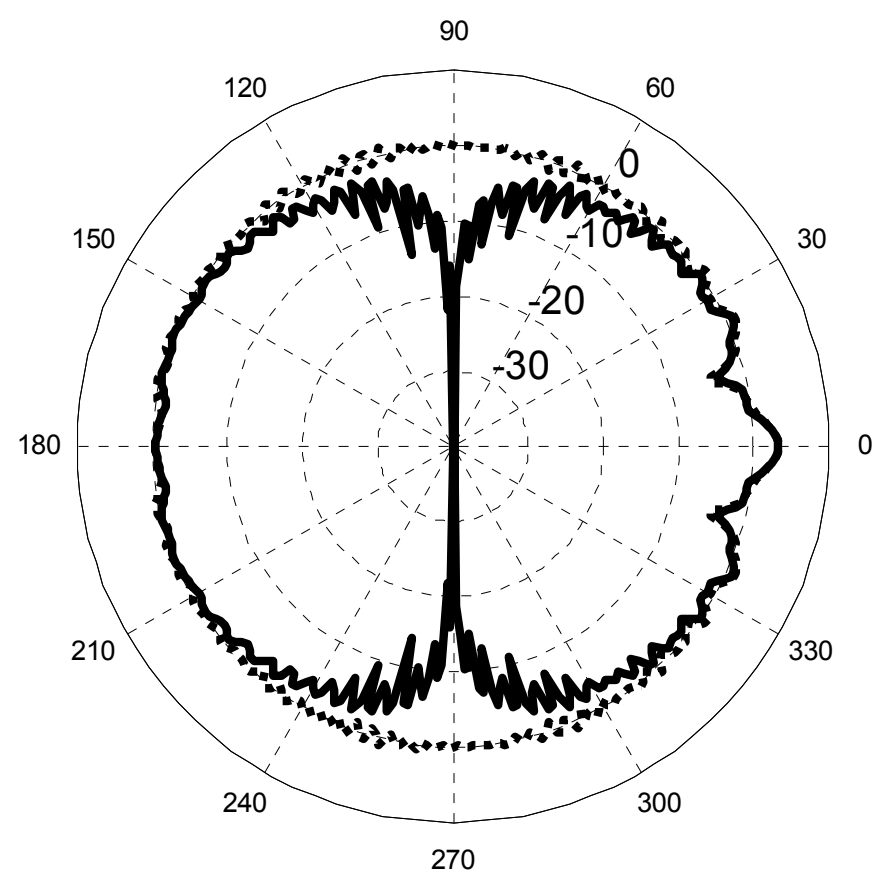

Figure 7.- Far field pattern of fields radiated by the dipole (left part of the plot) and the re-constructed by the 3D-CORPS (right part of the plot). 